\title{
The contact layer method in calculating of the shear compounds.
}

\author{
Vladimir Andreev ${ }^{1, *}$, Robert Turusov $^{1}$, and Nikita Tsybin $^{1}$ \\ ${ }^{1}$ Moscow state university of civil engineering, Yaroslavskoye shosse, 26, Moscow, Russia, 129337
}

\begin{abstract}
The paper presents a solution of the problem of the stress-strain state of a three-layer model under a shear load. The interaction between layers is accomplished by the contact layer in which the substances of adhesive and substrate are mixed. We will consider the contact layer as the transversal anisotropic medium with such parameters that it can be represented as a set of short elastic rods, which are not connected to each other. Three variants of the resolving system of equations are considered. For each of the variant, a calculation is made for the three load cases. The curves of distribution of the normal forces and tangential stresses in the contact layers were obtained.
\end{abstract}

\section{Statement of problem}

Consider the model of a multilayer beam (Fig. 1)

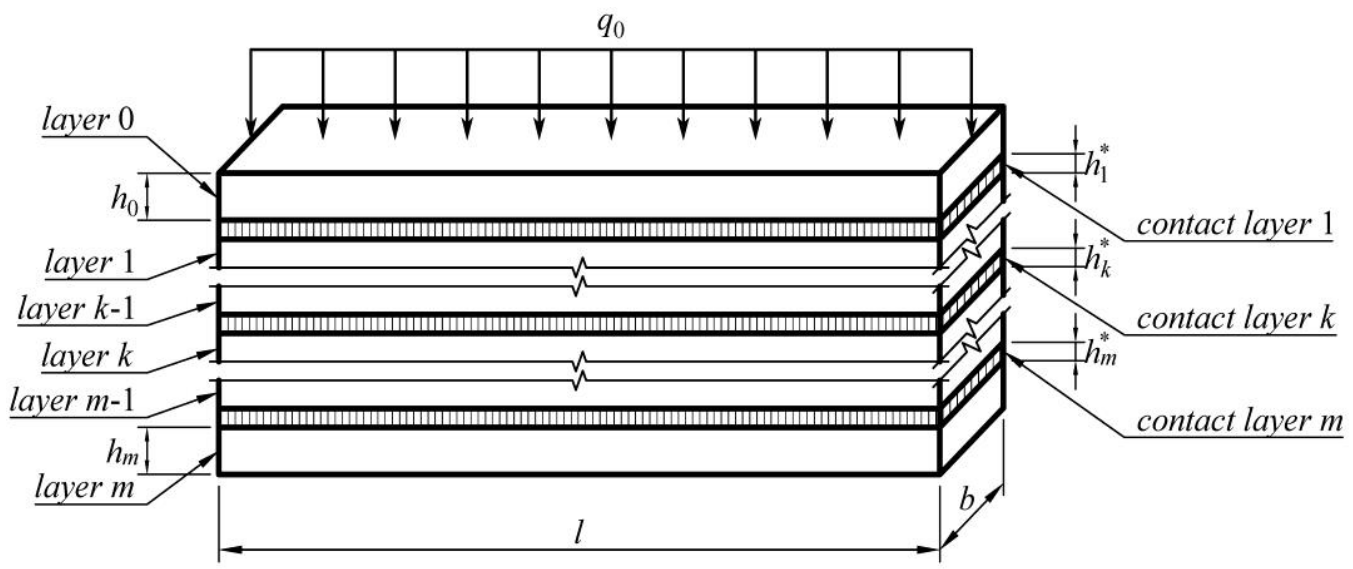

Fig. 1. The model of a multilayer beam.

The resolving equations for the external layers with taking into account the bending obtained in [1] are written below

\footnotetext{
* Corresponding author: asv@mgsu.ru
} 


$$
\left.\begin{array}{c}
\frac{d^{2} N_{k}}{d x^{2}}=\frac{d P_{x, k}^{t}}{d x}-\frac{d P_{x, k}^{b}}{d x} \\
-D_{k} \frac{d^{4} v_{k}}{d x^{4}}+\frac{h_{k}}{2}\left(\frac{d P_{x, k}^{t}}{d x}+\frac{d P_{x, k}^{b}}{d x}\right)=P_{z, k}^{t}-P_{z, k}^{b} .
\end{array}\right\}
$$

Disregarding of the bending will give

$$
\frac{d^{2} N_{k}}{d x^{2}}=\frac{d P_{x, k}^{t}}{d x}-\frac{d P_{x, k}^{b}}{d x}
$$

The values $P_{x, k}^{t}, P_{x, k}^{b}, P_{z, k}^{t}, P_{z, k}^{b}$ represent the external forces applied to the top and bottom faces of the external layers.

Consider the contact layer.

Because the rods are not connected, in Hooke's law for an orthotropic body Young's modulus $E_{x, k}^{*}$ and Poisson ratio $\mu_{k}^{*}$ must be set equal to zero. The symbol * will then be used to mark all values related to the contact layer.

The system of two differential equations with respect to the stresses in the contact layers can be obtained as a result of integration of the differential equations of equilibrium, with taking into account the continuity of the displacements of the external layers and contact layers.

$$
\left.\begin{array}{c}
\frac{\tau_{k}^{*}}{G_{k}^{*}}=\frac{\left(h_{k}^{*}\right)^{2}}{12 E_{k}^{*}} \frac{d^{2} \tau_{k}^{*}}{d x^{2}}+\frac{1}{2} \frac{d}{d x}\left(v_{t, k}^{*}+v_{b, k}^{*}\right)-\frac{1}{h_{k}^{*}}\left(u_{t, k}^{*}-u_{b, k}^{*}\right) ; \\
\sigma_{k}^{*}=-y_{k}^{*} \frac{d \tau_{k}^{*}}{d x}-\frac{E_{k}^{*}}{h_{k}^{*}}\left(v_{t, k}^{*}-v_{b, k}^{*}\right) .
\end{array}\right\}
$$

In these equations: $E_{y, k}^{*}=E_{k}^{*}, G_{x y, k}^{*}=G_{k}^{*}$. Values $v_{t, k}^{*}, v_{b, k}^{*}, u_{t, k}^{*}, u_{b, k}^{*}$ represent the displacements of the top and bottom external layers and are determined from expressions

$$
\begin{gathered}
u_{t, k}^{*}=u_{0, k-1}-\frac{h_{k-1}}{2} \frac{d v_{k-1}}{d x} ; u_{b, k}^{*}=u_{0, k}+\frac{h_{k}}{2} \frac{d v_{k}}{d x} ; \\
v_{t, k}^{*}=v_{k-1} ; v_{b, k}^{*}=v_{k} .
\end{gathered}
$$

The displacementы in the contact layer are a nonlinear function of the argument $y_{k}^{*}$ in this case, and the tangential stresses in the contact layer are a constant value.

In the case of a one-dimensional problem of the contact layer, the displacements are a linear function. As result, equations (3) taking form

$$
\left.\begin{array}{l}
\frac{\tau_{k}^{*}}{G_{k}^{*}}=-\frac{1}{h_{k}^{*}}\left(u_{t, k}^{*}-u_{b, k}^{*}\right) ; \\
\sigma_{k}^{*}=-\frac{E_{k}^{*}}{h_{k}^{*}}\left(v_{t, k}^{*}-v_{b, k}^{*}\right) .
\end{array}\right\}
$$

Simplifications used in (2) and (4) essentially simplify the solution of the problem; due to the fact that the number of unknown functions in the resolving system of differential equations is reduced. However, that simplifications lead to failure of the boundary conditions with respect to tangential stresses in the contact layer.

A three-layer model is considered for the calculations (Fig. 2) 


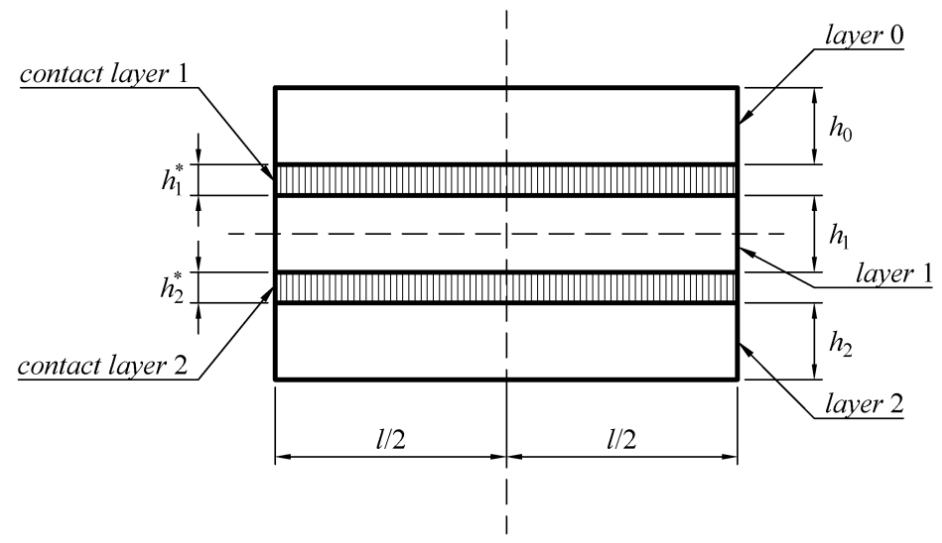

Fig. 2. Three-layer model.

This model is the most common in the calculation of adhesive compounds.

Three versions of the system of resolving equations are considered for comparison.

-Variant 1 represent a model that takes into account the bending of the external layers and the nonlinearity of the distribution of displacements in the contact layer. The resolving system of equations represented by relations (1) and (3) in this case.

-Variant 2 represent a model that takes into account the bending of the external layers and the linearity of the distribution of displacements in the contact layer. The resolving system of equations represented by relations (1) and (4) in this case.

-Variant 3 does not take into account the bending in the external and the nonlinearity of the distribution of displacements in the contact layer (2), (4).The resolving system of equations is represented by (2), (4). Some examples of calculations are given in $[2,3]$.

Below are the load cases of calculating for shear (Fig. 3).

1)

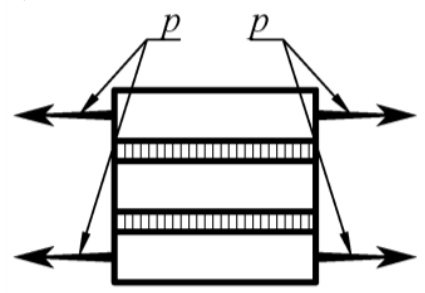

2)

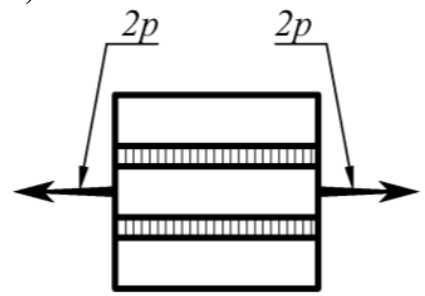

3)

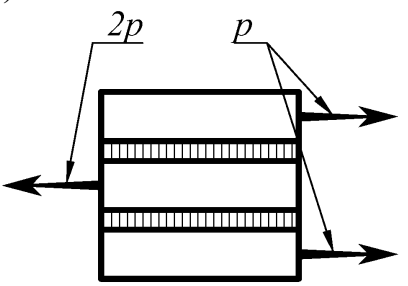

Fig. 3. Load cases.

Boundary conditions for axial forces are represented below

$$
\left.\begin{array}{l}
N_{0}\left(-\frac{l}{2}\right)=[p, 0, p] ; N_{0}\left(\frac{l}{2}\right)=[p, 0,0] ; \\
N_{1}\left(-\frac{l}{2}\right)=[0, p, 0] ; N_{1}\left(\frac{l}{2}\right)=[0, p, 2 p] ; \\
N_{2}\left(-\frac{l}{2}\right)=[p, 0, p] ; N_{2}\left(\frac{l}{2}\right)=[p, 0,0] .
\end{array}\right\}
$$


Boundary conditions for bending moment and shear force

$$
M\left(-\frac{l}{2}\right)=M\left(\frac{l}{2}\right)=0 ; Q\left(-\frac{l}{2}\right)=Q\left(\frac{l}{2}\right)=0 .
$$

Boundary conditions for shear stresses at the contact layer

$$
\tau_{1}^{*}\left(-\frac{l}{2}\right)=\tau_{1}^{*}\left(\frac{l}{2}\right)=0 ; \tau_{2}^{*}\left(-\frac{l}{2}\right)=\tau_{2}^{*}\left(\frac{l}{2}\right)=0 .
$$

In this case, taking into account the symmetry with respect to layer 1 , can obtain the following relations

$$
\begin{gathered}
N_{2}=N_{0} ; N_{1}=2\left(p-N_{0}\right) ; Q_{1}=M_{1}=0 ; \\
v_{2}=-v_{0} ; v_{1}=0 ; u_{2}=u_{0} ; D_{2}=D_{0} ; h_{2}=h_{0} ; \\
P_{x, 0}^{t}=P_{x, 2}^{b}=P_{z, 0}^{t}=P_{z, 2}^{b}=0 .
\end{gathered}
$$

Below are written the expressions that determine the stress state of the model.

$$
\left.\begin{array}{c}
\tau_{1}^{*}=-\frac{d N_{0}}{d x} ; \tau_{2}^{*}=-\tau_{1}^{*} ; \\
M_{0}=-D_{0} \frac{d^{2} v_{0}}{d x^{2}} ; M_{2}=-M_{0} ; \\
Q_{0}=-D_{0} \frac{d^{3} v_{0}}{d x^{3}}-\frac{h_{0}}{2} \tau_{1}^{*} ; Q_{2}=-Q_{0} .
\end{array}\right\}
$$

Analytical solutions of systems of equations 1-3 it is not possible to write down because they are cumbersome. We give only the calculation results for the following parameters.

$$
h_{0}=2 \mathrm{~mm} ; h_{1}=0.2 \mathrm{~mm} ; b=2 \mathrm{~mm} ; l=20 \mathrm{~mm} ; h^{*}=0.1 \mathrm{~mm} ;
$$

$$
E_{0}=2400 \mathrm{MPa} ; E_{1}=72000 \mathrm{MPa} ; E^{*}=1200 \mathrm{MPa} ; G^{*}=1200 \mathrm{MPa} \text {. }
$$

Those parameters correspond to the [4] Kargin model - the simplest model of unidirectional reinforced plastic consisting of two polymer plates (layers 0 and 2) and a fiber reinforcement layer (layer 1).

Below are curves represented calculation results for load case 1. Variants of resolving equations are marked with the numbers 1,2 , and 3 .

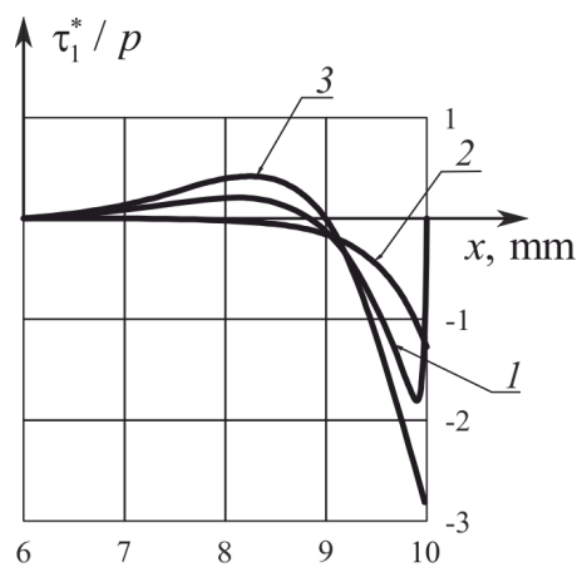

Fig. 5. Curves of tangential stresses in the contact layer 1 for the load case 1 .

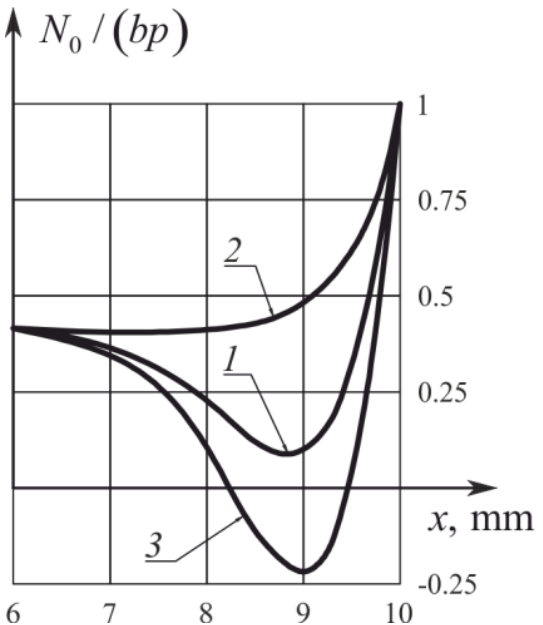

Fig. 6. Curves of axial force in the external layer 0 for the load case 1 . 
Below are curves represented calculation results for load case 2 .

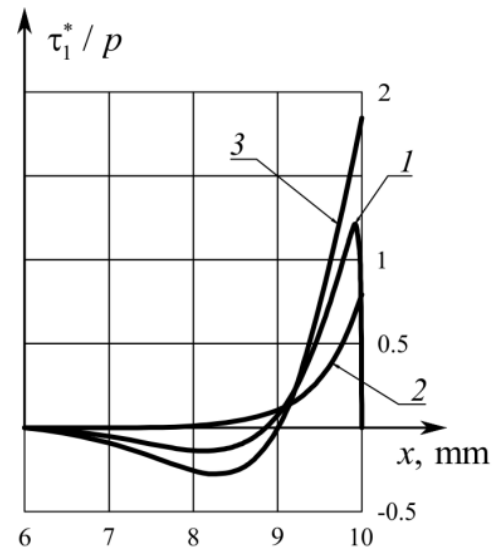

Fig. 7. Curves of tangential stresses in the contact layer 1 for the load case 2 .

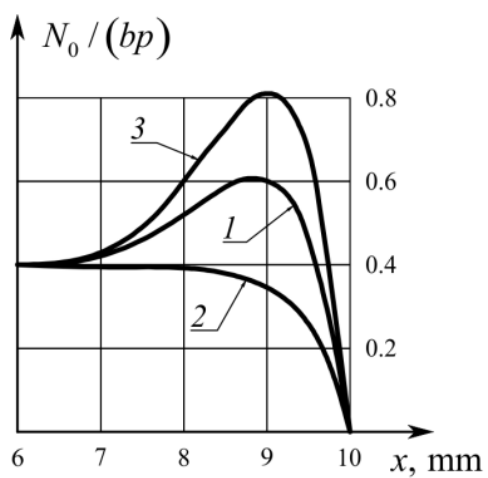

Fig. 8. Curves of axial force in the external layer 0 for the load case 2 .

For the load cases 1 and 2, the calculation results are given only for the right-hand side in view of the symmetry.

Below are curves represented calculation results for load case 3.

The results of the calculation for load case 3 are given for the left and right side of the model, since there is no symmetry.

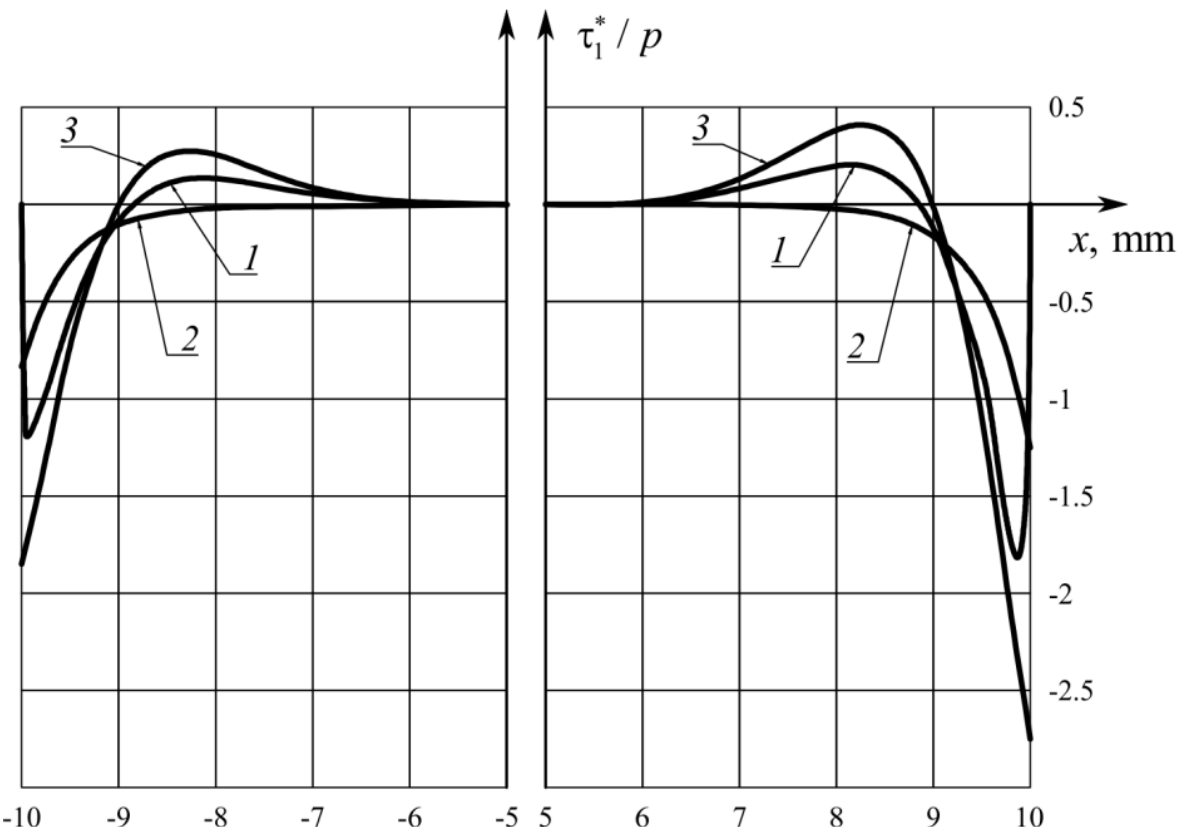

Fig. 9. Curves of tangential stresses in the contact layer 1 for the load case 3. 


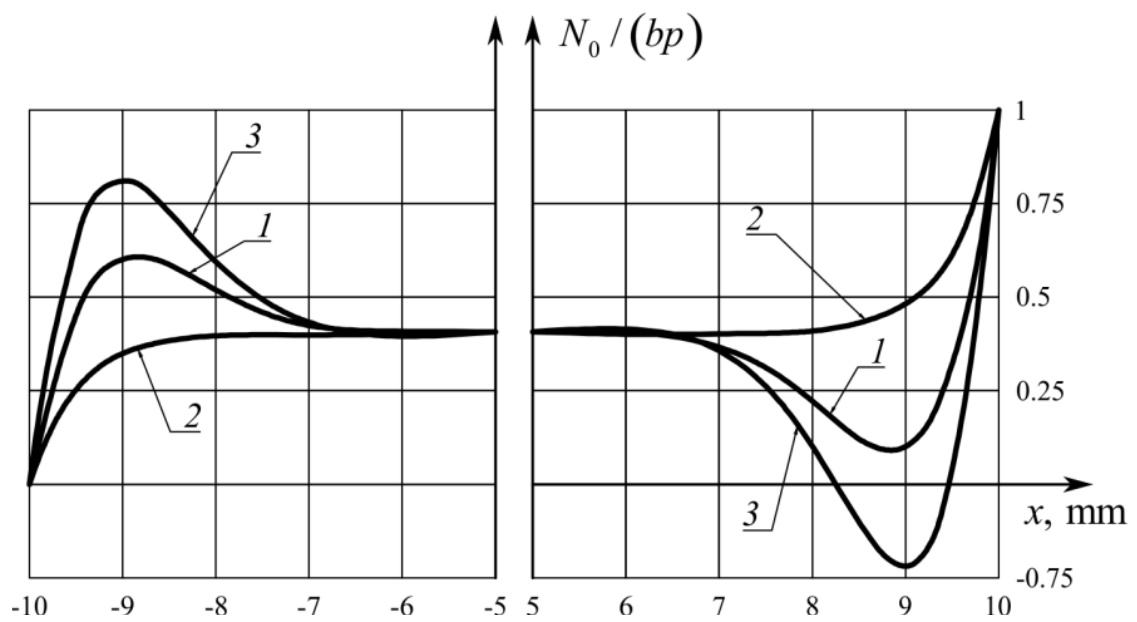

Fig. 10. Curves of axial force in the external layer 0 for the load case 3.

\section{Conclusions}

The results of the calculations show that variant 1 of resolving system of equations fulfills all the boundary conditions. However, it is not applicable for practical calculations, as result of its complexity.

Variant 2 can be used to calculate bending moments and shear forces, in models where these values are more important that axial forces and tangential stresses in the contact layer.

Variant 3 can be used to determine the zone of tangential stress concentration. Despite the fact that the value maximum of tangential stresses in this case is overestimated, it is the most suitable for practical calculations. Some results with using that model are given in paper [5].

Additional calculation models are given in paper [6].

\section{Acknowledgements}

This study was performed with the financial support of the RF Ministry of Education and Science (the basic part of the state task for 2017-2019).

\section{References}

1. Andreev V.I., Turusov R.A., Tsybin N.Yu. Application of the contact layer in the solution of the problem of bending the multilayer beam. Procedia Engineering. 2016. Vol. 153. P. 59-65.

2. Tsybin N.Yu., Turusov R.A., Andreev V.I. Comparison of creep in free polymer rod and creep in polymer layer of the layered composite. Procedia Engineering. 2016. Vol. 153. P. 51-58.

3. Andreev V.I., Turusov R.A., Tsybin N.Yu. Long strength of layered composite under normal fracture. Advances in Engineering Research, 2015. Vol. 39. P. 1703-1708. 
4. Turusov R.A. Elastic and thermal behavior of a layered structure. Part II. Calculation results and their analysis. Mechanics of Composite Materials. 2015. Vol. 51(1). P. 127134.

5. Turusov R.A., Kuperman A.M. Elastic properties of thin adhesive interlayers. Polymer Science. Series D. 2014. Vol. 7(1). P. 1-8.

6. Andreev V.I., Barmenkova E.V. The modeling of the real building object by using the model of a two layer beam of variable rigidity on an elastic basis. Applied Mechanics and Materials. 2012Vol. 204-208. P. 3596-3599. 